\title{
Cambios Físico-Químicos y Sensoriales Producidos por el Tipo de Corte y Empaque en Zanahoria (Daucus carota L.) Mínimamente Procesada
}

\author{
Saúl Dussán-Sarria(1), Carlos A. Garcia-Mogollon(2), Nelson Gutiérrez-Guzmán ${ }^{(3)}$ \\ (1) Univ. Nacional de Colombia - Sede Palmira, Fac. de Ingeniería y Administración, \\ A.A. 237. Palmira, Valle, Colombia (e-mail: sdussan@unal.edu.co). \\ (2) Univ. de Sucre, Fac. de Ingeniería, Cra 28 \# 5-267 Barrio Puerta Roja, Sincelejo, Sucre-Colombia \\ (3) Universidad Surcolombiana, Facultad de Ingeniería, Avenida Pastrana Borrero - Carrera 1, \\ Bloque de Ingeniería 2 Piso, Neiva, Huila-Colombia
}

${ }^{*}$ Autor a quien debe ser dirigida la correspondencia.

Recibido Sep. 12, 2014; Aceptado Nov. 28, 2014; Versión final recibida Jun. 18, 2014

\begin{abstract}
Resumen
En este estudio se evaluó el tipo de corte y de empaque de zanahorias frescas (Daucus carota L.) almacenadas a $9 \pm 2^{\circ} \mathrm{C}$ y $89 \%$ de humedad relativa durante 15 días, sobre los atributos de calidad de la hortaliza. Se evaluaron atributos físico-químicos, color y grado de satisfacción del consumidor. Se utilizó el delineamiento experimental completamente al azar con arreglo factorial compuesto por tres factores: forma de corte (cubo, rodaja), tipo de empaque (vacío, PET) y tiempo de almacenamiento con niveles $(0,3,6,9$, 12, 15 días), totalizando veinticuatro tratamientos. Se aplicó ANOVA y el test de Tukey y Dunnett para las notas sensoriales, empleado el software SPSS 15.0 para Windows. Todos los atributos mostraron diferencias significativas a través del periodo evaluado $(p<0.05)$. El tratamiento más adecuado de conservación bajo refrigeración de zanahoria mínimamente procesada fue al corte en forma de cubos y empacada al vacío.
\end{abstract}

\section{Physicochemical and Sensory Changes Produced by the Type of Cutting and Packing of Fresh-Cut Carrot}

\begin{abstract}

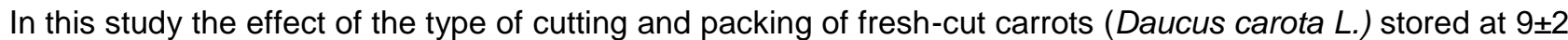
${ }^{\circ} \mathrm{C}$ and $89 \%$ of relative humidity during 15 days on quality attributes of the vegetable was evaluated. The physico-chemical attributes, color and subjective appreciation through a hedonic scale were evaluated. An experimental randomized design was used for the study with factorial array composed by three factors: cutting shape (cube, slice), type of packing (vacuum, PET) and the time of storage with levels of $0,3,6,9,12$ y 15 days, completing twenty four treatments. The Analysis of variance ANOVA was used and also the Tukey and Dunnett test $(p<0.05)$ to compare the sensory notes, employing the SPSS base software for Windows. All attributes showed significant differences through the evaluated period $(p<0.05)$. The most suitable treatment of conservation of fresh-cut carrot in cold storage was carrot cut in cubes and vacuum packed.
\end{abstract}

Keywords: carrot, Daucus carota, vegetables, quality, conservation, fresh-cut 


\section{INTRODUCCIÓN}

La tecnología de procesamiento mínimo de productos hortofrutícolas se encuentra en auge. Los productos se procesan para proveer al consumidor de un alimento listo para consumir con beneficios para la salud por su aporte en sustancias fitoquimicas (Escobar et al., 2014a). Esta tendencia ha impulsado a nuevas presentaciones de los vegetales cuarta gama (mezclas de hortalizas cortadas o frutas mínimamente procesadas) para complementar nutrientes como las vitaminas, minerales y antioxidantes (Santos et al., 2012) o aprovechar las características de uno solo. En particular, la zanahoria se destaca por ser un producto rico en antioxidantes, destacando-se los antioxidantes nutricionales como la vitamina $A$, vitamina $C$ y vitamina $\mathrm{E}$, y una gran cantidad de antioxidantes no nutricionales, como compuestos b-carotenos y polifenoles (Luciano et al., 2009)

Se debe considerar que durante el pelado y troceado se aumenta la superficie de tejido haciéndolo más susceptible a alteraciones microbianas; además, del incremento de la tasa respiratoria, la producción de etileno y el metabolismo de los compuestos fenólicos que aceleran la senescencia y el deterioro de los productos hortofrutícolas (Ramos et al., 2013). El procesamiento mínimo de vegetales frescos utiliza técnicas que se combinan sinérgicamente limitando el crecimiento microbiano, las reacciones físicas, químicas y bioquímicas. Algunas de estas técnicas son el pre-enfriamiento, la refrigeración en toda la cadena, la atmosfera modificada (AM) y la atmosfera controlada (AC) (Sanjinez-Argandoña et al., 2010). Han sido usadas en la conservación de zanahoria y lechuga cortada almacenada a $4^{\circ} \mathrm{C}$ usando el color como indicador (Morales, 2011).

Se tienen diversos estudios de aplicación de atmosferas modificadas para conservar zanahoria mínimamente procesada (Mastromatteo et al., 2012). Simões et al. (2010) indican que zanahorias mínimamente procesadas pierden rápidamente su color anaranjado brillante, el desarrollo de un aspecto blanquecino o rubor blanca en la superficie, reduciendo así la aceptabilidad de los consumidores y Emmambux y Minnaar (2003) observaron que la decoloración causada por la deshidratación del tejido y el deterioro microbiano son los principales problemas que limitan la vida útil de las zanahorias recién cortadas, sin embargo también se hace necesario explorar la incidencia del tipo de corte aplicado a la zanahoria en la decoloración.de esta. La comercialización de zanahoria mínimamente procesada puede ser una alternativa de mercado interesante al producto fresco dado su alto uso en ensaladas. El objetivo de este trabajo fue evaluar en el procesamiento mínimo el efecto que tiene el tipo de corte, el material de empaque y el tiempo de almacenamiento en los atributos físico-químicos y sensoriales de la zanahoria y a partir de los análisis definir la forma más adecuada de conservación.

\section{MATERIALES Y MÉTODOS}

\section{Procesamiento mínimo}

Las zanahoria frescas fueron lavadas con solución de cloro a $200 \mu \mathrm{l}^{-1}$, para la eliminación de residuos provenientes del campo y desinfección. Luego, se retiraron las extremidades, la cáscara o piel y se procesaron a dos cortes: cubos $(\mathrm{Cu})$ y rodajas (Ro); los cortes fueron sometidos a una inmersión en solución de cloro a $150 \mu^{\mathrm{I}^{-1}}\left(15^{\circ} \mathrm{C}\right.$ y $\left.6,5 \mathrm{pH}\right)$ y posterior enjuague con solución de cloro a $5 \mu \mathrm{I} \mathrm{I}^{-1}\left(20^{\circ} \mathrm{C}\right.$ y 7,5 $\mathrm{pH}$ ) con duración total de 5 minutos. Fue retirando el exceso de agua de los cortes de zanahoria. Se acondicionaron los cortes en cajas polietileno tereftalato PET de $12 \mathrm{~cm} \times 8 \mathrm{~cm} \times 4 \mathrm{~cm}$ y al vacío (Va) utilizando bolsa de polietileno lineal de baja densidad PEBD de espesor de $70 \mu \mathrm{m}$ de $19 \mathrm{~cm} \times 14 \mathrm{~cm}$. Las respectivas permeabilidades son: cajas PET al $\mathrm{CO}_{2}$ de $112,3392 \mathrm{~cm}^{3} \mathrm{~m}^{-2}$ día-1 y al O de $3945,2929 \mathrm{~cm}^{3} \mathrm{~m}^{-2}$ día- ${ }^{-1}$ y bolsas de PEBD calibre $70 \mu \mathrm{m}$ para aplicar vacío al $\mathrm{CO}_{2}$ de $6264,8897 \mathrm{~cm}^{3} \mathrm{~m}^{-2}$ día ${ }^{-1}$ y al O $\mathrm{O}_{2}$ de $1163,4795 \mathrm{~cm}^{3} \mathrm{~m}^{-2} \mathrm{día}^{-1}$. Con excepción de la zanahoria cortada acondicionada al vacío, los cortes acondicionados en PET iniciaron en atmosfera modificada pasiva. El almacenamiento se realizó en un cuarto frio a $9 \pm 2^{\circ} \mathrm{C}$ y 85 a $90 \%$ de HR simulando las condiciones de exposición en el supermercado, durante el cual se evaluaron atributos físico-químicos y sensoriales desde el inicio del almacenamiento y cada 3, 6, 9,12 y 15 días.

\section{Atributos analíticos}

El contenido de Sólidos Solubles (SS) medidos en ${ }^{\circ}$ Brix se determinaron según la norma NTC 4624 (ICONTEC, 1999a), los valore de pH según la norma NTC 4592 (ICONTEC, 1999b) y los valores de Acidez Titulable (AT) de acuerdo a la norma NTC 4623 (ICONTEC, 1999c). El análisis de color se realizó determinando las coordenadas de color del espacio CIELab. Las mediciones se hicieron tomando imágenes fotográficas con una videocamara Sony Handycam Dcr-Sr47 Hd 60 Gb Zoom 60x, y analizadas en el software PhotoShop 7.0. A partir de estos parámetros de color se calcularon las magnitudes 
psicofísicas croma $\left(\mathrm{C}^{*} \mathrm{ab}\right)$ y tono $(\mathrm{Hab})$ y el parámetro total de color $(\Delta \mathrm{E})$, utilizando las siguientes ecuaciones:

$C^{*} a b=\sqrt{a^{*}+b^{* 2}}$

$H^{*} a b=\arctan \frac{a^{*}}{b^{*}}$

$\Delta E^{*} a b=\sqrt{\Delta L^{*}+\Delta a^{*}+\Delta b^{* 2}}$

El índice de blanqueamiento (WI) fue determinado acorde a la siguiente ecuación (Bolin y Huxsoll, 1991):

$W I=100-\left[\left(100-L^{*}\right)^{2}+a^{* 2}+b^{* 2}\right]^{1 / 2}$

\section{Atributos sensoriales}

La evaluación sensorial se efectuó desde el inicio y hasta el final del período de almacenamiento para evaluar la apariencia general de las zanahorias frescas cortadas. Se utilizó una escala estructurada de grado de satisfacción, donde: $1=$ Me gusta muchísimo; $2=$ Me gusta mucho; $3=$ Me gusta moderadamente; $4=\mathrm{Me}$ gusta ligeramente; $5=\mathrm{Ni}$ gusta ni disgusta; $6=\mathrm{Me}$ disgusta ligeramente; $7=\mathrm{Me}$ disgusta moderadamente; $8=$ Me disgusta mucho y $9=$ Me disgusta muchísimo. Se escogieron 30 jueces consumidores con edades entre 20 y 30 años.

\section{Diseño experimental}

Se aplicó un diseño experimental completamente al azar con un arreglo factorial compuesto por tres factores: la forma de corte de las zanahorias con dos niveles (cubo, rodaja) y el tipo de empaque (vacío, PET); y el tiempo de almacenamiento con niveles $(0,3,6,9,12,15$ días), para un total de 24 tratamientos. Fueron realizadas tres repeticiones. Los tratamiento fueron designados así: Ro-Va: Rodajas al vacío, RoPET: Rodajas en PET, Cu-Va: Cubos al vacío y Cu-PET: cubos en PET. Fue realizado ANOVA empleando el software SPSS 15.0 para Windows (SPSS Inc., Chicago, Illinois). Para determinar entre que niveles del factor fueron significativas las diferencias se aplicó el Test de Tukey con un nivel de significancia de p<0,05. Se aplicó un test de Dunnett para comparar las notas sensoriales en los periodos 3, 6, 9, 12 y 15 con respeto al periodo inicial.

\section{RESULTADOS Y DISCUSIÓN}

\section{Cambios en los atributos físico-químicos}

En la Tabla 1 se observan los valores de los atributos físico-químicos ( ${ }^{\circ} \mathrm{Brix}, \mathrm{pH}$ y AT) de la zanahoria fresca cortada (ZFC) en cubos y rodajas empacadas al vacío y en cajas PET almacenadas por 15 días a $9 \pm 2^{\circ} \mathrm{C}$ y $89 \%$ de HR.

No se presentó diferencia significativa $(p>0,05)$ en los tratamientos sobre la variable acidez y si hubo un efecto significativo $(p<0,05)$ del tiempo. En todos los tratamientos hubo una relación directa entre el aumento de los valores de $\mathrm{pH}$ en el tiempo y consecuente disminución de los valores de AT.

Para todos los tratamientos evaluados hasta el día 06 del almacenamiento se presentó un ligero descenso de los valores de SS de $8{ }^{\circ}$ Brix a 5,6 ${ }^{\circ}$ Brix en promedio y posterior a este día un ligero aumento hasta alcanzar al final del almacenamiento un valor promedio de $6,8^{\circ} \mathrm{Brix}$. La poca variación de los valores de los SS entre el inicio y el final del almacenamiento es debida a la poca actividad respiratoria típica de productos no climatéricos y los efectos positivos del uso del frio durante el almacenamiento (Kader, 2002; Chitarra y Chitarra, 2005). El tiempo en almacenamiento tiene un efecto significativo en los ${ }^{\circ}$ Brix de la zanahoria $(p=$ $0.0001)$ al igual que el tipo de empaque $(p=0.03919)$, en cambio la forma en cubos y rodajas no mostro efectos significativos $(p=0.5458)$, similares resultados reportaron Alegria et al. (2010) en zanahorias rayadas tratadas con 200ppm de cloro por $1 \mathrm{~min}$, Pushkala et al. (2012) en zanahorias cortadas en empaques de polietileno de baja densidad perforado. 
Tabla 1: Características físico-químicas de la zanahoria fresca cortada.

\begin{tabular}{|c|c|c|c|c|c|c|c|}
\hline Día & Tratamiento & \multicolumn{2}{|c|}{${ }^{\circ}$ Brix } & \multicolumn{2}{|c|}{$\mathrm{pH}$} & \multicolumn{2}{|c|}{ AT } \\
\hline \multirow{4}{*}{0} & Ro-Va & 8,00 & $\pm 0,001$ & 5,87 & $\pm 0,010$ & 0,23 & $\pm 0,003$ \\
\hline & $\mathrm{Cu}-\mathrm{Va}$ & 8,00 & $\pm 0,001$ & 5,88 & $\pm 0,010$ & 0,24 & $\pm 0,004$ \\
\hline & Ro-PET & 8,00 & $\pm 0,002$ & 5,87 & $\pm 0,000$ & 0,23 & $\pm 0,003$ \\
\hline & CU-PET & 8,00 & $\pm 0,001$ & 5,88 & $\pm 0,010$ & 0,24 & $\pm 0,001$ \\
\hline \multirow{4}{*}{3} & Ro-Va & 6,00 & $\pm 0,001$ & 6,52 & $\pm 0,000$ & 0,07 & $\pm 0,001$ \\
\hline & Cu-Va & 6,00 & $\pm 0,002$ & 5,59 & $\pm 0,000$ & 0,07 & $\pm 0,002$ \\
\hline & Ro-PET & 7,00 & $\pm 0,001$ & 5,83 & $\pm 0,000$ & 0,07 & $\pm 0,001$ \\
\hline & CU-PET & 6,00 & $\pm 0,002$ & 5,59 & $\pm 0,000$ & 0,03 & $\pm 0,002$ \\
\hline \multirow{4}{*}{6} & Ro-Va & 5,75 & $\pm 0,350$ & 6,06 & $\pm 0,660$ & 0,07 & $\pm 0,001$ \\
\hline & $\mathrm{Cu}-\mathrm{Va}$ & 5,50 & $\pm 0,000$ & 5,59 & $\pm 0,000$ & 0,03 & $\pm 0,002$ \\
\hline & Ro-PET & 6,00 & $\pm 0,000$ & 6,09 & $\pm 0,000$ & 0,07 & $\pm 0,000$ \\
\hline & CU-PET & 5,00 & $\pm 0,000$ & 6,15 & $\pm 0,000$ & 0,01 & $\pm 0,001$ \\
\hline \multirow{4}{*}{9} & Ro-Va & 6,00 & $\pm 0,000$ & 7,06 & $\pm 0,000$ & 0,07 & $\pm 0,002$ \\
\hline & $\mathrm{Cu}-\mathrm{Va}$ & 6,00 & $\pm 0,000$ & 7,00 & $\pm 0,000$ & 0,05 & $\pm 0,001$ \\
\hline & Ro-PET & 6,00 & $\pm 0,000$ & 6,17 & $\pm 0,000$ & 0,06 & $\pm 0,005$ \\
\hline & CU-PET & 7,00 & $\pm 0,000$ & 6,29 & $\pm 0,000$ & 0,02 & $\pm 0,000$ \\
\hline \multirow{4}{*}{12} & Ro-Va & 6,50 & $\pm 0,710$ & 7,12 & $\pm 0,080$ & 0,07 & $\pm 0,001$ \\
\hline & $\mathrm{Cu}-\mathrm{Va}$ & 6,00 & $\pm 0,000$ & 6,29 & $\pm 1,020$ & 0,05 & $\pm 0,001$ \\
\hline & Ro-PET & 7,00 & $\pm 1,410$ & 6,25 & $\pm 0,060$ & 0,06 & $\pm 0,006$ \\
\hline & CU-PET & 7,00 & $\pm 0,000$ & 6,38 & $\pm 0,130$ & 0,03 & $\pm 0,003$ \\
\hline \multirow{4}{*}{15} & Ro-Va & 7,00 & $\pm 0,000$ & 7,17 & $\pm 0,000$ & 0,03 & $\pm 0,000$ \\
\hline & $\mathrm{Cu}-\mathrm{Va}$ & 6,00 & $\pm 0,000$ & 6,57 & $\pm 0,000$ & 0,02 & $\pm 0,000$ \\
\hline & Ro-PET & 7,00 & $\pm 0,000$ & 6,29 & $\pm 0,000$ & 0,01 & $\pm 0,001$ \\
\hline & CU-PET & 7,00 & $\pm 0,000$ & 6,29 & $\pm 0,000$ & 0,01 & $\pm 0,000$ \\
\hline
\end{tabular}

Considerando los valores del pH de la ZFC en todos los tratamientos durante el almacenamiento refrigerado exhibieron se presentó una variación de 5,875 a 6,580 , es decir es un producto altamente propenso al ataque de microorganismos de ahí la importancia de la higienización más cuando se trata de un producto mínimamente procesado. El análisis de varianza mostró diferencias estadísticamente significativas para el $\mathrm{pH}$ durante tiempo de almacenamiento, tipo de corte y el tipo de empaque $(p<0,05)$. La acidez titulable expresada como porcentaje de ácido málico varió de $0,23 \%$ a 0,01 \% excepto en la zanahoria en rodajas empacada en PET que vario hasta $0,12 \%$, manteniéndose en descenso hasta el sexto día de evaluación. El motivo de éste comportamiento es debido al descenso en la producción de ácidos orgánicos utilizados también en el proceso respiratorio del tejido vegetal (Chitarra y Chitarra, 2005). El incremento en azúcares simples y la disminución de ácidos orgánicos en el tejido vegetal involucra reacciones enzimáticas favorecidas por el daño físico, estos cambios afectan la relación dulce/ácido que determina el sabor del producto, el lixiviado por el corte puede generar pérdidas hasta del $60 \%$ de azucares, ácidos y demás compuestos (Alegria et al., 2009).

\section{Caracterización del color}

En la caracterización colorimétrica de la zanahoria mínimamente procesada las magnitudes psicofísicas croma $\left(\mathrm{C}_{\mathrm{ab}}\right)$, tono $\left(\mathrm{H}_{\mathrm{ab}}\right)$ y el parámetro total de cambio de color $(\Delta \mathrm{Eab})$ mostraron un descenso a lo largo del periodo de almacenamiento (Tabla 2).

La magnitud psicofísica croma $\left(\mathrm{C}_{\mathrm{ab}}\right)$ mostro diferencias significativas para el tiempo de almacenamiento y la forma de corte $(p=0,0001 ; p=0,0134)$, no para el tipo de empaque $(p=0,5198)$. El tono $\left(H_{a b}\right)$ es influenciado por el tiempo de almacenamiento y el tipo de empaque $(p=0,0001)$ caso contrario con la forma de corte que tiene un efecto no significativo $(p=0,0806)$. El parámetro total de cambio de color $(\Delta \mathrm{E} a b)$ presenta diferencias significativas $(p=0,0423)$ para la forma de corte, en el caso de las otras fuentes de variación no presenta diferencias significativas. Dado que $\Delta \mathrm{Eab}$ es el resultado de la combinación de las diferentes coordenadas $\left(L^{*}, a^{*}, b^{*}\right)$, esta representa la magnitud de la diferencia en el color pero no indica la dirección de esta diferencia. La zanahoria contiene principalmente $\alpha$ - y $\beta$-carotenoides y la reducción del $C_{a b}$ está asociada a la perdida de estos pigmentos. Pushkala et al. (2012) reportaron reducción del $C_{a b}$ a los 10 días a $10^{\circ} \mathrm{C}$ de zanahorias cortadas. Las ZFC empacadas al vacío mostraron menor pérdida del color que las empacadas en PET, corroborando que a bajo nivel de oxigeno la degradación de los pigmentos es lenta. 
Tabla 2: Valores de caracterización colorimétrica de la zanahoria fresca cortada.

\begin{tabular}{|c|c|c|c|c|c|c|c|c|c|}
\hline \multirow[t]{2}{*}{ Día } & \multirow{2}{*}{$\begin{array}{l}\text { Tratamiento } \\
\text { Ro-Va }\end{array}$} & \multicolumn{2}{|c|}{$L^{*}$} & \multicolumn{2}{|c|}{ Cab } & \multicolumn{2}{|c|}{$\mathrm{Hab}$} & \multicolumn{2}{|c|}{$\Delta \mathrm{Eab}$} \\
\hline & & 66,68 & $\pm 1,12$ & 60,03 & $\pm 0,06$ & 0,77 & $\pm 0,004$ & 65,42 & $\pm 0,02$ \\
\hline \multirow{3}{*}{0} & Cu-Va & 66,68 & $\pm 1,12$ & 60,23 & $\pm 0,40$ & 0,77 & $\pm 0,004$ & 65,42 & $\pm 0,02$ \\
\hline & Ro-PET & 66,68 & $\pm 1,12$ & 60,19 & $\pm 0,33$ & 0,77 & $\pm 0,004$ & 65,42 & $\pm 0,02$ \\
\hline & Cu-PET & 66,68 & $\pm 1,12$ & 60,00 & $\pm 6,87$ & 0,77 & $\pm 0,004$ & 65,43 & $\pm 4,75$ \\
\hline \multirow{4}{*}{3} & Ro-Va & 63,23 & $\pm 0,40$ & 57,07 & $\pm 1,74$ & 0,70 & $\pm 0,005$ & 63,44 & $\pm 1,13$ \\
\hline & $\mathrm{Cu}-\mathrm{Va}$ & 63,93 & $\pm 0,12$ & 59,00 & $\pm 0,98$ & 0,73 & $\pm 0,003$ & 63,78 & $\pm 0,94$ \\
\hline & Ro-PET & 63,43 & $\pm 0,40$ & 57,60 & $\pm 1,39$ & 0,73 & $\pm 0,005$ & 61,80 & $\pm 2,08$ \\
\hline & Cu-PET & 64,10 & $\pm 0,17$ & 55,20 & $\pm 0,35$ & 0,75 & $\pm 0,001$ & 63,20 & $\pm 0,00$ \\
\hline \multirow{4}{*}{6} & Ro-Va & 59,97 & $\pm 1,38$ & 54,55 & $\pm 0,04$ & 0,62 & $\pm 0,005$ & 62,56 & $\pm 0,05$ \\
\hline & $\mathrm{Cu}-\mathrm{Va}$ & 62,76 & $\pm 0,42$ & 56,93 & $\pm 0,12$ & 0,69 & $\pm 0,003$ & 60,31 & $\pm 0,01$ \\
\hline & Ro-PET & 59,16 & $\pm 1,38$ & 43,13 & $\pm 0,03$ & 0,72 & $\pm 0,000$ & 54,01 & $\pm 0,01$ \\
\hline & Cu-PET & 62,76 & $\pm 0,42$ & 51,15 & $\pm 2,55$ & 0,69 & $\pm 0,003$ & 57,66 & $\pm 3,20$ \\
\hline \multirow{4}{*}{9} & Ro-Va & 54,87 & $\pm 0,23$ & 50,17 & $\pm 2,52$ & 0,65 & $\pm 0,002$ & 59,67 & $\pm 1,63$ \\
\hline & $\mathrm{Cu}-\mathrm{Va}$ & 56,93 & $\pm 0,12$ & 54,93 & $\pm 1,10$ & 0,72 & $\pm 0,003$ & 63,56 & $\pm 1,88$ \\
\hline & Ro-PET & 58,50 & $\pm 0,50$ & 54,87 & $\pm 6,80$ & 0,69 & $\pm 0,002$ & 58,79 & $\pm 2,77$ \\
\hline & Cu-PET & 62,10 & $\pm 0,17$ & 53,13 & $\pm 0,23$ & 0,73 & $\pm 0,006$ & 62,65 & $\pm 0,05$ \\
\hline \multirow{4}{*}{12} & Ro-Va & 53,44 & $\pm 0,30$ & 46,47 & $\pm 0,40$ & 0,68 & $\pm 0,001$ & 60,54 & $\pm 0,04$ \\
\hline & $\mathrm{Cu}-\mathrm{Va}$ & 55,56 & $\pm 1,62$ & 53,05 & $\pm 0,04$ & 0,71 & $\pm 0,003$ & 64,03 & $\pm 0,03$ \\
\hline & Ro-PET & 61,10 & $\pm 0,17$ & 55,86 & $\pm 0,57$ & 0,74 & $\pm 0,002$ & 57,64 & $\pm 0,66$ \\
\hline & Cu-PET & 62,12 & $\pm 1,20$ & 55,54 & $\pm 1,25$ & 0,74 & $\pm 0,000$ & 62,26 & $\pm 0,20$ \\
\hline \multirow{4}{*}{15} & Ro-Va & 48,00 & $\pm 0,98$ & 43,85 & $\pm 1,66$ & 0,59 & $\pm 0,002$ & 63,73 & $\pm 1,87$ \\
\hline & $\mathrm{Cu}-\mathrm{Va}$ & 50,70 & $\pm 1,53$ & 50,44 & $\pm 1,47$ & 0,66 & $\pm 0,002$ & 58,38 & $\pm 3,27$ \\
\hline & Ro-PET & 59,69 & $\pm 0,53$ & 48,18 & $\pm 0,07$ & 0,73 & $\pm 0,001$ & 57,24 & $\pm 0,04$ \\
\hline & Cu-PET & 55,46 & $\pm 2,20$ & 47,74 & $\pm 4,50$ & 0,62 & $\pm 0,002$ & 59,81 & $\pm 1,42$ \\
\hline
\end{tabular}

El índice de blanqueamiento (WI) establece el grado de decoloración de la zanahoria durante el almacenamiento, mayores valores de WI significan mayor grado de decoloración. Según sea el grado de pérdida de color puede significar defecto de calidad que el consumidor asocia a pérdida de frescura. La zanahoria mínimamente procesada en rodajas presentó un WI inicial de 27,52 y al final del periodo de almacenamiento un valor de 34,08 cuando empacadas al vacío a un valor de 39,13 cuando empacadas en PET. Cuando cortada en cubos el valor WI final fue de 28,42 empacada al vacío y de 36,81 empacada en PET, similares resultados fueron reportados por varios autores (Alegria et al., 2010).

La ZFC en cubos y empacada al vacío presentó el menor grado de blanqueamiento y la ZFC en rodajas y empacada en PET el mayor grado de blanqueamiento. El relativo aumento de WI durante el almacenamiento, es decir mayor grado de decoloración, se relaciona con la deshidratación de la superficie debida al corte y asociada con la formación de compuestos fenólicos y lignina influenciada por la enzima PAL (fenilalanina amonioliasa), lo cual puede ser prevenido manteniendo la superficie húmeda (Vargas et al., 2009). En la Figura 1 se observa que los valores de la relación de índice de blanqueamiento WI/Wlo son afectados por el tipo de empaque y el periodo de almacenamiento $(p<0,05)$.

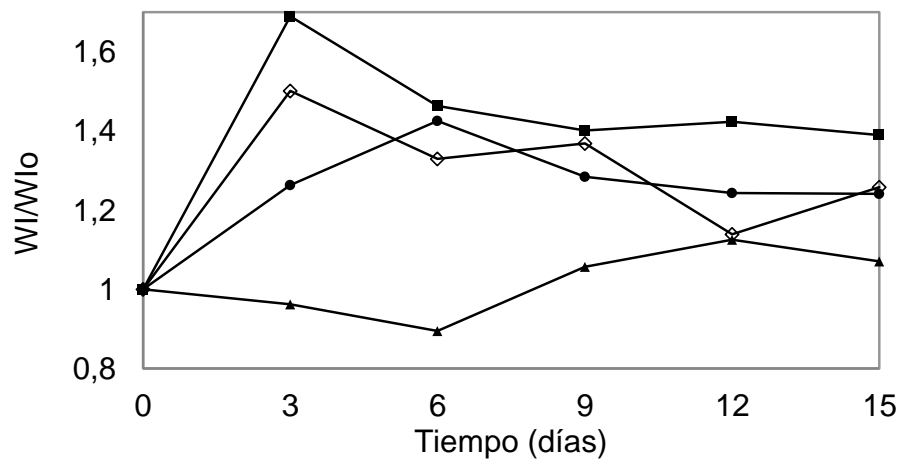

Fig. 1: Variación de los valores de la relación de índice de blanqueamiento (WI/Wlo) de zanahoria mínimamente procesada y almacenada durante 15 dias a temperatura de $9 \pm 2{ }^{\circ} \mathrm{C}$ y 85 a $90 \%$ de HR. •: Rodajas al vacío, a: Rodajas PET, $\boldsymbol{\Delta}$ : Cubos vacío y $\diamond$ : cubos PET. 
En este trabajo se evidenció efectivamente que el grado de deshidratación y por ende de la perdida de color de la zanahoria fue mayor en el cote rodajas por presentar una mayor área superficial la cual queda expuesta a un mayor grado de pérdida de humedad en relación al corte en cubo.

\section{Grado de satisfacción del consumidor}

Mediante la aplicación del test de Dunnett el grado de satisfacción (SG) de los consumidores no expresaron diferencias significativas $(p<0.05)$ en los períodos $3,6,12$ y 15 con respecto al primer día. Sin embargo, para los diferentes tratamientos si existieron diferencias significativas al $95 \%$ estableciendo dos grupos, uno formado por Cu-PET y el otro por los restantes tratamientos (Figura 2). Es importante destacar que durante los 15 días de evaluación no se observó presencia de microrganismos y las notas sensoriales estuvieron entre "Me gusta moderadamente" (3) y "Me gusta ligeramente" (4). La ZFC en cubos y empacada en PET (Cu-PET) obtuvo la peor nota de 4,04 $\pm 0,24$ y La ZFC en cubos y empacada al vacío (Cu-Va) exhibió la mejor nota sensorial al final del experimento, con un valor de $3,19 \pm 0,34$, correspondiente a la escala entre me gusta moderadamente y me gusta ligeramente. La mejor aceptación de este tratamiento se confirma con el menor grado de blanqueamiento exhibido. Similar resultado obtuvieron mejor aceptabilidad por tratamiento con recubrimiento comestible en zanahorias mínimamente procesadas conservando su calidad sensorial general (Escobar et al., 2014b) y Das Neves et al. (2011) observaron decremento en el color durante el almacenamiento sin afectar la aceptación por parte de los consumidores.

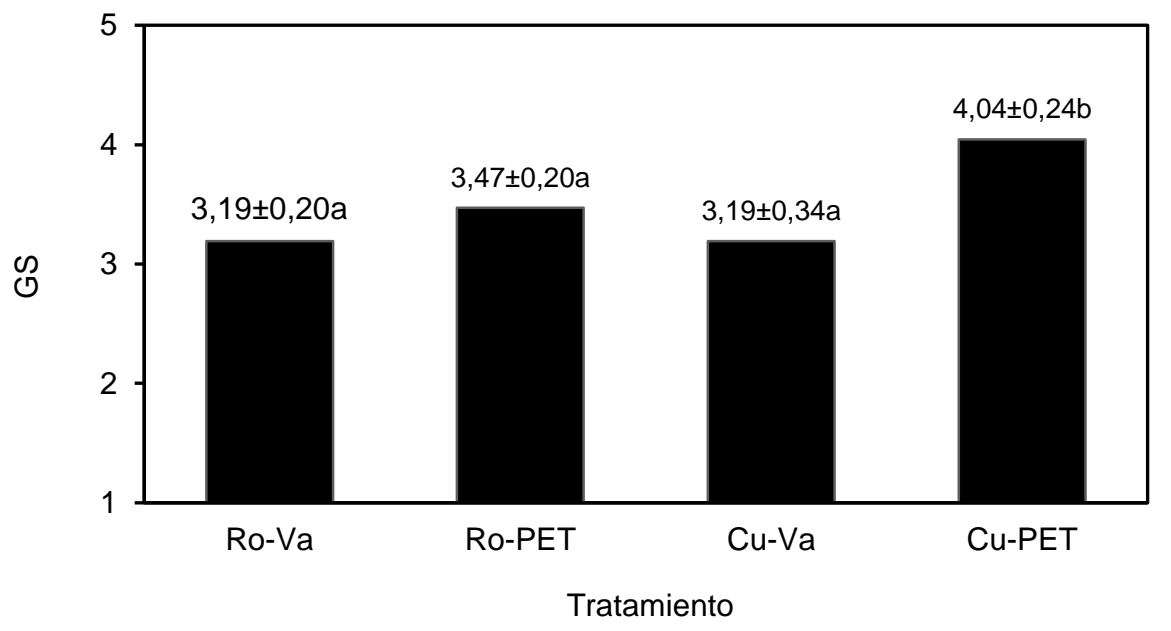

Fig. 2: Grado de satisfacción (GS) medio de zanahoria fresca cortada y almacenada durante 15 días a temperatura de $9 \pm 2^{\circ} \mathrm{C}$ y $89 \%$ de humedad relativa

Las propiedades visuales de la zanahoria fresca cortada es un parámetro importante que el consumidor tomo en cuenta para evaluar su calidad. El tiempo de almacenamiento no tuvo un efecto significativo en la satisfacción del consumidor ( $p=0,9761)$, si tuvo un efecto el tipo de corte y el tipo de empaque $(p=0,001)$. De acuerdo a Abe et al. (1993) y Abe y Chachin (1995) la tasa de deterioro de la zanahoria cortada es influenciada por el tamaño de las secciones, por la proporción relativa del área vertical/horizontal y por el tipo de tejido. La frecuencia de consumo de zanahoria fresca cortada está aumentando progresivamente siendo el tipo de corte el factor principal que hace la diferencia en la preferencia (Resende et al., 2004). El color es uno de los atributos importantes en la calidad del alimento. Aunque el color no necesariamente refleja el valor nutricional o funcional, este determina la aceptabilidad del producto por el consumidor.

Cruz et al. (2006) reportaron que la aceptabilidad de ZFC se correlaciona con los niveles más alto de $\mathrm{CO}_{2}$ dentro de los envases, menor firmeza y una mayor pérdida de electrolitos. La decoloración en la superficie del tejido (blanqueo de la superficie) fue la causa principal de la baja aceptabilidad, la cual es debida a la deshidratación del tejido, lo cual también pudo provocar un aumento en la firmeza causado por el endurecimiento y lignificación del tejido. Estudios previos han mostrado que éstos son los principales problemas que presenta la ZFC (Gorny et al., 2000).

\section{CONCLUSIONES}

El tratamiento agroindustrial más adecuado que proporcionó la mejor conservación de los atributos de calidad a la zanahoria mínimamente procesada se refiere al corte en forma de cubos y empacada al vacío. En esta condición y bajo refrigeración a $9 \pm 2^{\circ} \mathrm{C}$ y 85 a $90 \%$ de HR se conserva bien durante 15 días. 
El tipo de corte tiene influencia directa en el grado de decoloración de la zanahoria mínimamente procesada. El corte en rodaja por presentar una mayor área superficial y mayor pérdida de humedad presenta un grado de decoloración mayor que el corte en cubo.

En zanahoria mínimamente procesada refrigerada, el tiempo de almacenamiento, forma de corte, el tipo de empaque afecta el contenido de ácidos orgánicos reduciéndolos y aumentando los valores de $\mathrm{pH}$.

El color y la aceptación sensorial de la zanahoria mínimamente procesada a lo largo del periodo de almacenamiento es influenciado por el tipo de corte y el tipo de empaque.

\section{REFERENCIAS}

Abe, K., Yoshimura, K. y Iwata, T. Effect of cutting direction on storability and phisiological changes in partially processed carrots. Journal of the Japanese Society for Food Science and Technology, 40(2):101$106(1993)$

Abe, K. y Chachin, K. Physiological properties of carrot sections having difference in ratio of vertical or transverse cut surface. Journal of Japanese Society of Cold Preservation of Foods, 21(2): 87-92 (1995)

Alegria, C., Pinheiro, J., Gonçalves, E., Fernandes, I., Moldão, M. y Abreu, M. Quality attributes of shredded carrot (Daucus carota L. cv. Nantes) as affected by alternative decontamination processes to chlorine. Innovative Food Science and Emerging Technologies, 10: 61-69 (2009)

Alegria, C., Pinheiro, J., Gonçalves, E., Fernandes, I., Moldão, M. y Abreu, M. Evaluation of a pre-cut heat treatment as an alternative to chlorine in minimally processed shredded carrot. Innovative Food Science and Emerging Technologies, 11: 155-161 (2010)

Bolin, H. y Huxsoll, C. Control of minimally processed carrot (Daucus carota) surface discoloration caused by abrasion peeling. Journal of Food Science, 56(2): 416-418 (1991)

Chitarra, M. y Chitarra, A. Pós-colheita de frutos e hortaliças: fisiologia e manuseio, Lavras: ESAL/FAEPE, segunda edición, Lavras: UFLA, Brasil. 783 (2005)

Cruz, S., Acedo, E., Díaz, M., Islas, M. y González, G. Efectividad de Sanitizantes en la Reducción Microbiana y Calidad de Zanahoria Fresca Cortada. Revista Fitotecnia Mexicana, 299-306 (2006)

Das Neves, L., Dias, R. y Rodriguez, A. Influence of temperature and edible coating on the physical and chemical parameters and sensory acceptance of fresh-cut organic carrots Influencia de la temperatura y cobertura comestible en los parámetros físicos y químicos y aceptación sensorial de las zanahorias cortadas frescas, doi: 10.1080/19476330903515464, Journal of food, 9(1): 31-36 (2011)

Emmambux, N. M. y Minnaar, A. The effect of edible coatings and polymeric packaging films on the quality of minimally processed carrots, doi: 10.1002/jsfa.1509, Journal of the Science of Food and Agriculture, 83: 1065-1071 (2003)

Escobar, A., Márquez, C., Restrepo, C. y Pérez, L. Aplicación de tecnología de barreras para la conservación de mezclas de vegetales mínimamente procesados. Rev.Fac.Nal.Agr.Medellín 67(1): 7237$7245(2014 a)$

Escobar, A., Márquez, C., Restrepo, C., Cano, J. y Patiño, J. Aplicación de tratamiento térmico, recubrimiento comestible y baño químico como tratamientos poscosecha para la conservación de hortalizas mínimamente procesadas, doi: http://dx.doi.org/10.15446/acag.v63n1.40149, Acta Agronómica. 63 (1): 1-10 (2014b)

Gorny, J., Cifuentes, R., Hess-pierce, B. y Kader, A. Quality changes in fresh-cut pear slices as affected by cultivar, ripeness stage, fruit size, and storage regime. J. Food Sci. 65: 541-544 (2000)

ICONTEC Instituto Colombiano de Normas Técnicas y Certificación NTC 4624. Jugo de frutas y hortalizas. Determinación del contenido de sólidos solubles. Método refractimétrico. pp 11. Bogota-Colombia (1999a)

ICONTEC Instituto Colombiano de Normas Técnicas y Certificación NTC 4592. Productos frutas y verduras determinación de pH. pp 12. Bogota-Colombia (1999b) 
ICONTEC Instituto Colombiano de Normas Técnicas y Certificación. NTC 4623. Productos frutas y verduras determinación de la acidez titulable. pp 13. Bogota-Colombia (1999c)

Luciano, J. Q., Isabel, O. S., Robert, S. F., Afonso, M. R. y Olga, M. B. Comparative study on antioxidant properties of carrot juice stabilized by highintensity pulsed electric field or heat treatments, doi: 10.1002/jsfa.3767, Journal of the Science of Food and Agriculture, 89: 2636-2642 (2009)

Mastromatteo, M., Conte, A., Del Nobile, M.A. Packaging strategies to prolong shelf life of fresh carrots (Daucus carota L.), doi:10.1016/j.ifset.2011.10.010, Innov. Food Sci. Emerg. Technol. 13, 215-220 (2012)

Morales, C. Determinación de indicadores de calidad por apariencia para vegetales frescos precortados, Tesis de Magister, Facultad de Ingenierías, Universidad de la Sabana, Chía, Colombia (2011)

Pushkala, R., Parvathy, K. y Srividya, N. Chitosan powder coating, a novel simple technique for enhancement of shelf life quality of carrot shreds stored in macro perforated LDPE packs, doi:10.1016/j.ifset.2012.03.003, Innovative Food Science and Emerging Technologies, 16: 11-20 (2012)

Ramos, B., Miller, F., Brandão, T., Teixeira, P. y Silva, C. Fresh fruits and vegetables-An overview on applied methodologies to improve its quality and safety, doi:10.1016/j.ifset.2013.07.002, Innovative Food Science and Emerging Technologies, 20: 1-15 (2013)

Resende, J., Coelho, A., Castro, E., Saggin júnior, O., Nascimento, T. y Benedetti, B. Modificações sensoriais em cenoura minimamente processada e armazenada sob refrigeração doi: 10.1590/S010205362004000100031, Horticultura Brasileira, Brasília, 22(1): 147-150 (2004)

Sanjinez-Argandoña, E. J., Branco, I. G., Takito, S. Y., y Corbari, J. Influencia de la deshidratación osmótica y de la adición de cloruro de calcio en la conservación de kiwis minimamente procesados, http://dx.doi.org/10.1590/S0101-20612010000500031, Food Science and Technology (Campinas), 30, 205$209(2010)$

Santos, M.I., A. Cavaco, J. Gouveia, M.R. Novais, P. J. Nogueira, L. Pedroso and M.A. Ferreira. Evaluation of minimally processed salads commercialized in Portugal, doi:10.1016/j.foodcont.2011.06.022, Food Control 23(1): 275-281 (2012)

Simões A. N.; Ventrella M. C.; Moretti C. L.; Carnelossi, M. A. G.; Puschmann R. Anatomical and physiological evidence of white blush baby carrot surfaces, doi:10.1016/j.postharvbio.2009.07.007, Postharvest Biology and Technology. 55: 45-52 (2010)

Vargas, M.; Chiralt, A.; Albors, A.; Gonzalez- Martinez, C. Effect of chitosan-based edible coatings applied by vacuum impregnation on quality preservation of fresh-cut carrot, doi:10.1016/j.postharvbio.2008.07.019, Postharvest Biology and Technology, 51: 263-271 (2009) 